\title{
Lipoprotein(a) apheresis in patients with peripheral arterial disease: rationale and clinical results
}

\author{
Norbert Weiss ${ }^{1}$ - Ulrich Julius ${ }^{2}$
}

Published online: 15 March 2019

(c) The Author(s) 2019

\begin{abstract}
Patients with symptomatic peripheral arterial disease (PAD) are at a very high risk of cardiovascular morbidity and mortality. Elevated lipoprotein(a) levels have been shown to be a risk factor for coronary artery disease (CAD) and stroke. More recently elevated lipoprotein(a) levels have also been demonstrated to be associated with prevalent and incident PAD, and even may be a stronger risk factor for PAD compared with CAD. Lipoprotein apheresis is currently the only efficient way to lower lipoprotein(a) levels. Lipoprotein(a) apheresis has been shown to reduce major coronary events in patients with CAD. There is increasing evidence that lipoprotein(a) apheresis also reduces the rate of major adverse limb events such as peripheral revascularizations and amputations in PAD patients, and improves symptoms of PAD such as pain on exertion. This review summarizes the current knowledge on the clinical role of lipoprotein(a) for PAD and the disease-specific effect of lipoprotein(a) apheresis, and suggests indications for screening for and treating of elevated lipoprotein(a) levels in patients with $\mathrm{PAD}$.
\end{abstract}

Keywords Peripheral arterial disease $\cdot$ Intermittent claudication $\cdot$ Critical limb ischemia $\cdot$ Atherosclerosis · Cardiovascular prevention

\section{Introduction}

Peripheral arterial disease (PAD), coronary artery disease (CAD) and cerebrovascular disease (CVD) are different manifestations of atherosclerotic vascular disease. The whole spectrum of atherosclerotic cardiovascular diseases shares common cardiovascular risk factors such as smoking, diabetes, hypertension and hyperlipidemia and require identical risk factor management for primary and secondary prevention [1]. The term PAD is not well defined and sometimes used to summarize diseases of all extracranial and extracoronary arteries including carotid artery disease, mesenteric and renal artery disease, as well as upper and lower extremities arterial disease [2]. Most often, and also in this paper, the term PAD is used synonymously to lower

This article is part of the special issue "Lp(a) - Update 2018"

Norbert Weiss

norbert.weiss@ukdd.de

1 University Center for Vascular Medicine and Department of Medicine III-Section Angiology, University Hospital Carl Gustav Carus, Technische Universität Dresden, Fetscherstr. 74, 01307 Dresden, Germany

2 Department of Medicine III-Lipidology and Center for Extracorporeal Treatment, University Hospital Carl Gustav Carus, Technische Universität Dresden, Dresden, Germany extremities arterial disease, the most frequent manifestation of PAD [3, 4].

The fate of patients with PAD is determined by the prognosis of the affected extremity and by the total cardiovascular prognosis. Patients with intermittent claudication rarely progress to critical limb ischemia and are at low risk for amputation, which is estimated to be at 2 to $4 \%$ within 5 years after initial presentation. These patients, however, are ten times more likely to suffer a non-fatal or fatal cardiac or cerebrovascular event [5]. Patients that initially present with critical limb ischemia, i.e. with rest pain and/or ischemic foot lesions, are a different entity with both a very high one year mortality and amputation rate up to $30 \%$ each.

Besides the above mentioned predominant risk factors elevated levels of lipoprotein(a) have been identified as an important additional atherothrombotic agent that increases the risk of developing PAD and adversely influences the prognosis of patients with established PAD patients quoad extremitatem and quoad vitam [6]. This review will summarize and discuss current knowledge about the association and putative causal role of lipoprotein(a) for PAD and the effect of lipoprotein(a) lowering by lipoprotein apheresis and PAD itself and overall cardiovascular prognosis of PAD patients. 


\section{Peripheral arterial disease is a marker for very high cardiovascular risk}

Current guidelines consider clinically manifest PAD as a risk equivalent to manifest $\mathrm{CAD}$ requiring identical treatment strategies and goals with respect to atherosclerotic risk factor management [1]. The overall cardiovascular risk associated with PAD, however, still seems to be underestimated as there is undertreatment of PAD compared with CAD patients with lipid-lowering, anti-hypertensive and antiplatelet drugs [7]. Recent data show that patients with symptomatic PAD even without concomitant CAD carry a higher risk of death and cardiovascular events compared with patients with CAD without clinically manifest PAD.

Pereg and colleagues [8] prospectively followed a cohort of nearly 10,000 patients for up to 10 years (mean follow-up 5.6 \pm 2.3 years) who had undergone their first clinically driven coronary intervention (82.8\% of patients) or peripheral vascular intervention (17.2\% of patients). The PAD patients had a significantly worse long-term prognosis with a significantly increased all-cause mortality compared with the CAD patients (unadjusted hazard ratio 2.95, multivariately adjusted hazard ratio 1.86). This observation cannot be solely explained by undertreatment of PAD patients. Randomized controlled trials of novel lipid-lowering or antiplatelet drugs, in which PAD and CAD patients were included and in which all patients received optimal conventional treatment, confirmed the higher cardiovascular risk of PAD patients.

The "Further Cardiovascular Outcomes Research with PCSK9 Inhibition in patients with Elevated Risk" (FOURIER) trial was a randomized trial of the proprotein convertase subtilisin/kexin type 9 (PCSK9) inhibitor evolocumab versus placebo in nearly 28,000 patients with stable atherosclerotic vascular disease who were on optimal medical therapy including high-dose statin. The trial included 3642 patients with symptomatic PAD (1505 with no prior myocardial infarction or stroke) and 23,922 patients with prior myocardial infarction of stroke without known PAD [9]. In the placebo group, the 2.5-year event rate of the composite endpoint of cardiovascular death, myocardial infarction, stroke, hospitalization for unstable angina, or coronary revascularization was significantly higher in patients with symptomatic PAD (16.8\%) compared with patients without PAD (12.1\%, adjusted hazard ratio 1.57).

"Prevention of Cardiovascular Events in Patients with Prior Heart Attack Using Ticagrelor Compared to Placebo on a Background of Aspirin-Thrombolysis in Myocardial Infarction 54" (PEGASUS TIMI 54) was a trial of dual antiplatelet therapy using ticagrelor in stable post-myocardial infarction (MI) patients. All patients received optimal medical treatment of cardiovascular risk factors. The placebo group included 6633 post-MI patients without PAD and
404 post-MI patients who also had PAD [10]. During three years of follow-up the composite endpoint of cardiovascular death, myocardial infarction and stroke was significantly more frequent in the patients with PAD (19.3\%) compared with the patients without PAD (8.4\%, unadjusted hazard ratio 2.46 , adjusted hazard ratio 1.6 ).

These two recent randomized trials emphasize the higher ischemic risk in patients with symptomatic PAD with and without concomitant $\mathrm{CAD}$ compared to patients with $\mathrm{CAD}$ without PAD even in patients who are under optimal conventional medical treatment. This suggests that the higher atherosclerotic burden in PAD compared with CAD and/or differences in the impact of different atherosclerotic risk factors in different manifestations of the disease may be responsible for the higher cardiovascular event rate in PAD patients.

\section{Elevated lipoprotein(a) levels are a risk factor for peripheral arterial disease}

Several case-control studies proposed an association between elevated lipoprotein(a) levels and the risk of suffering from PAD.

In the "Linz Peripheral Arterial Disease" (LIPAD) study from Lower Austria 213 patients with symptomatic PAD were matched to 213 controls for sex, age, and presence of diabetes [11]. Lipoprotein(a) concentrations were measured by a double-antibody enzyme-linked immunosorbent assay (ELISA), and apolipoprotein(a) isoform by sodium dodecyl sulfate (SDS) agarose gel electrophoresis under reducing conditions. Low molecular weight isoforms were defined as at least one allele with $\leq 22$ kringle-IV repeats. PAD patients compared with controls showed significantly higher medium lipoprotein(a) serum concentrations and a higher frequency of low molecular weight apolipoprotein(a) phenotype. After adjustment for potential confounding factors, lipoprotein(a) concentrations above the 75th percentile of the entire cohort were a significant predictor of PAD with an odds ratio of 3.73 , as was the low molecular weight apolipoprotein(a) phenotype (odds ratio 2.21). This association was consistently confirmed in several other case-control or cross sectional cohort studies in different populations including in total more than 10,000 subjects. The "Cardiovascular Disease in Intermittend Claudiation" (CAVASIC) and the "Kooperative Gesundheitsforschung in der Region Augsburg" (KORA F3 and KORA F4) studies included populations from southern Germany [12], a further cohort was studied in France [13], and the "MultiEthnic Study of Atherosclerosis" (MESA) included nearly 5000 European Americans, African Americans, Hispanic Americans and Chinese Americans [14]. 
The causality of elevated lipoprotein(a) for the development of PAD was then strongly suggested by three prospective cohort studies.

A substudy of the "European Prospective Investigation into Cancer and Nutrition" prospective population study (EPIC-Norfolk) included 25,639 female and male inhabitants of Norfolk, United Kingdom, aged between 29 and 79 years [15]. The baseline survey was performed between 1993 and 1997. Patients were followed until 2008. Outcomes were identified by linking a unique National Health Service number in the EPIC database with the East Norfolk Health Authority database. Mortality and hospitalizations for PAD and CAD were classified according to the respective International Classification of Diseases (ICD) codes. During 212,981 person-years at risk 2365 CAD and 596 PAD events occurred. Study participants in the highest sex-specific lipoprotein(a) quartile had an about 2-fold increased hazard ratio for the developing of PAD compared to study participants in the other lipoprotein(a) quartiles. The hazard ratio remained largely unchanged after adjusting for several putative confounding factors including low-density lipoprotein(LDL)-cholesterol. Notably, the predictive value of lipoprotein(a) levels in the highest quartile were higher for PAD (adjusted hazard ratio 2.06) compared with CAD (adjusted hazard ratio 1.33).

The latter finding is consistent with data from the prospective "Scottish Heart Health Extended Cohort" [16]. This large prospective cohort recruited 15,737 healthy men and women across Scotland between 1984 and 1995, and followed them through 2009 for CAD and PAD diagnoses. The 20-year risk for PAD hospitalization and mortality was significantly determined by baseline lipoprotein(a) levels with an hazard ratio of 1.21. In contrast, baseline lipoprotein(a) levels were not significantly predictive for CAD risk in this cohort.

The above mentioned findings although apply to populations from other geographic origin, as baseline lipoprotein(a) levels were also predictive for incident PAD during 6-year follow-up in a sample of more than 1000 Italian subjects over the age of 60 [17].

Taken together the above mentioned data strongly suggest that elevated lipoprotein(a) levels increase the risk of developing clinical relevant PAD that leads to hospitalization and mortality. Elevated lipoprotein(a) levels seem to be a more important risk factor for PAD compared with CAD (or stroke, the latter data not shown).

\section{Prognostic impact of elevated lipoprotein(a) levels in patients with peripheral arterial disease}

In patients with established symptomatic PAD elevated lipoprotein(a) levels also seem to be a risk factor for subsequent arterial events. Sanchez Monoz-Torreo et al. [18] prospectively followed a Spanish cohort of 1503 outpatients with symptomatic PAD for a mean of 36 months. During that time patients suffered from 122 myocardial infarctions, 118 strokes, 58 lower limb amputations, and 85 deaths. This event rate again indicates that patients with established PAD are at a very high cardiovascular and mortality risk. According to baseline lipoprotein(a) levels patients were separated into tertiles. Patients having lipoprotein(a) levels in the highest tertile above $50 \mathrm{mg} / \mathrm{dL}$ compared with patients in the lowest tertile below $30 \mathrm{mg} / \mathrm{dL}$ were shown to have an adjusted hazard ratio of $23.22(95 \%$ confidence interval 12.17-44.29) for suffering a myocardial infarction, of 64.52 (95\% confidence interval 29.13-142.93) for suffering a stroke, of 22.75 (95\% confidence interval 9.25-55.97) for suffering a lower limb amputation, and of 32.92 (95\% confidence interval 21.35-50.77) for any of these arterial events. These findings have to be confirmed in additional cohorts but indicate the high ongoing atherothrombotic risk of elevated lipoprotein(a) levels in patients with established atherosclerotic vascular disease.

\section{Efficacy of lipoprotein(a) apheresis in patients with peripheral arterial disease}

A causal relationship between elevated lipoprotein(a) levels and PAD that is strongly suggested by prospective cohort studies will finally be proven after lipoprotein(a) lowering therapies have shown to reduce the incidence and progression of PAD and other cardiovascular events in PAD patients. There is no effective pharmacological treatment to lower lipoprotein(a) levels clinically available at the moment, although RNA-targeted therapeutics to reduce lipoprotein(a) levels already have successfully completed phase II clinical trials [19]. Lipoprotein apheresis remains the only treatment option for patients with elevated lipoprotein(a) and progressive cardiovascular disease despite optimal medical treatment of all conventional cardiovascular risk factors. Lipoprotein apheresis has been shown to reduce the rate of cardiovascular events in patients with established coronary artery disease associated with high lipoprotein(a) levels [20-23].

Published data on the efficacy of lipoprotein apheresis in patients with PAD and elevated lipoprotein(a) are sparse, although PAD is highly prevalent in patients that are treated by lipoprotein(a) apheresis. Among 90 patients who started 
lipoprotein apheresis due to progressive cardiovascular disease and either isolated elevated lipoprotein(a) levels or a combination of elevated lipoprotein(a) and low-density lipoprotein cholesterol levels between 2008 and 2013 peripheral arterial disease was present in 44 and $53 \%$ of patients, respectively [24]. Among the 170 patients in the "Prospective Lipoprotein(a) Apheresis Registry Pro(a)Life" that represented around $60 \%$ of the German patients that were treated by lipoprotein(a) apheresis in 2011 [21] 30 lower limb peripheral arterial events had occurred in the two years before initiation of lipoprotein apheresis.

The latter registry followed these 170 patients and compared the cardiovascular event rates before and after initiation of lipoprotein(a) apheresis. In addition to a significant reduction of major cardiovascular events including myocardial infarction, percutaneous coronary intervention and coronary artery bypass grafting from 144 in the two years before to 31 in the two years after initiation of lipoprotein apheresis the rate of peripheral arterial events including peripheral arterial angioplasty, stenting or bypass surgery was reduced from 30 to 11, respectively. Furthermore, other non-coronary interventions such as carotid artery stenting or surgery or renal artery angioplasty or stenting was reduced from 7 to 1 , respectively [21].

In a monocentric, retrospective, longitudinal cohort study 35 patients with isolated lipoprotein(a) elevation had been included, and the rate of cardiovascular events in the $6.8 \pm 5.6$ years before and $6.8 \pm 4.9$ years after initiation of chronic lipoprotein apheresis was compared. Besides a $90.4 \%$ reduction of major adverse cardiac events including cardiac death, non-lethal myocardial infarction, coronary bypass surgery and percutaneous coronary interventions a reduction of peripheral vascular events from 11 in the years before to 2 in the years after initiation of lipoprotein apheresis could be observed [25].

Poller and coworkers [26, 27] prospectively followed 10 patients who were treated by lipoprotein apheresis due to symptomatic PAD that required a revascularization procedure, had elevated lipoprotein(a) levels above $60 \mathrm{mg} / \mathrm{dL}$ and were on sufficient treatment of all other cardiovascular risk factors. Outcome measures were the rate of clinically driven revascularizations in the 12 months prior compared with the 24 months after initiation of lipoprotein apheresis as well as the ankle-brachial blood pressure index, the walking distance determined by a standardized treadmill test, as well as subjective grading of the mean pain level during everyday physical activity on a 10-point visual analogue scale (the higher the score the worse the pain). Consistent with the above mentioned study, the study also showed a significant reduction of clinical driven revascularizations from 35 procedures during 120 patient-months in the year before to 2 procedures per 229 patient-months in the two years after initiation of lipoprotein apheresis. Lipoprotein apheresis also led to a rapid and sustained improvement of PAD symptoms in all patients. This is indicated by a significant reduction of the pain level from $7.0 \pm 1.5$ points at baseline to $1.6 \pm 0.7$ after one and $1.1 \pm 0.4$ after two years of lipoprotein apheresis. Consistently, the mean walking distance increased from $87 \pm 60 \mathrm{~m}$ at baseline gradually to $402 \pm 119$ after two years. This went along with a significant increase in the resting ankle-brachial index in the affected leg.

Taken together these data show that lipoprotein apheresis rapidly and significantly improves symptoms of PAD such as pain during physical activity and the walking distance and reduces the rate of clinically driven peripheral revascularizations.

\section{Conclusions}

Elevated lipoprotein(a) levels have been shown to be a risk factor for the development of cardiovascular disease in the coronary and cerebrovascular circulation. Although randomized controlled trials are missing and won't be possible to perform due to ethical reasons within the near future longitudinal observational studies strongly suggest that the reduction of lipoprotein(a) levels by lipoprotein apheresis significantly and clinical relevantly reduces the incidence of cardiovascular events [20-23, 25]. There is increasing evidence from case-control studies, cross-sectional cohort studies and longitudinal prospective cohort studies that elevated lipoprotein(a) levels are a risk factor for the development of PAD and for a worse clinical outcome in PAD patients [11-18]. Furthermore, longitudinal observational studies indicated that lipoprotein apheresis also reduces the necessity for clinically driven revascularizations in patients with PAD [21, 23, 25-27]. Whether or not it also reduces the extremely high burden of overall cardiovascular morbidity and mortality in PAD patients that is higher compared with patients with isolated CAD $[8,9]$ has not been studied so far but seems to be biologically plausible.

Therefore it is good clinical practice that patients with PAD and progressive disease despite control of all conventional risk factors should be screened for elevated lipoprotein(a) levels. In addition patients with elevated lipoprotein(a) levels and coronary or cerebrovascular atherosclerosis should also be screened for the presence of PAD, either symptomatic or asymptomatic, as patients with polyvascular disease in the coronary and peripheral circulation are at higher risk compared to patients with isolated CAD [10].

PAD patients with elevated lipoprotein(a) levels and progressive disease as indicated by recurrent clinical events requiring revascularization or progression of the disease stage from asymptomatic to intermittent claudication or critical limb ischemia or from intermittent claudication to critical 
limb ischemia even without the necessity or technical ability for revascularization should be considered candidates for lipoprotein apheresis. In addition patients with elevated lipoprotein(a) levels and clinical overt coronary and cerebrovascular disease that are found to develop PAD should be considered as patients with progressive atherosclerotic vascular disease and considered candidates for lipoprotein apheresis [28].

Although randomized controlled trials have not been performed and therefore have not proven the clinical efficacy of lipoprotein apheresis this treatment option cannot be withheld in high risk patients based on current evidence. To broaden the knowledge on the efficacy of lipoprotein apheresis in patients with atherosclerotic cardiovascular disease including PAD and elevated lipoprotein(a) levels, however, it is reasonable and seems to be mandatory to include all patients that initiate lipoprotein apheresis in prospective clinical trials or registries such as the "European Multicenter Study on the Effect of Lipoprotein(a) Elimination by Lipoprotein Apheresis on Cardiovascular Outcomes" (MultiSelect) [29].

Conflict of interest N. Weiss received speaker's honoraria from Fresenius Medical Care. U. Julius received speaker's honoraria from Kaneka, Diamed, Fresenius Medical Care. Both serve in the adjudication committee of the MultiSelect trial sponsored by Kaneka.

Open Access This article is distributed under the terms of the Creative Commons Attribution 4.0 International License (http:// creativecommons.org/licenses/by/4.0/), which permits unrestricted use, distribution, and reproduction in any medium, provided you give appropriate credit to the original author(s) and the source, provide a link to the Creative Commons license, and indicate if changes were made.

\section{References}

1. Catapano AL, Graham I, De Backer G, Wiklund O, Chapman MJ, Drexel H, Hoes AW, Jennings CS, Landmesser U, Pedersen TR, Reiner Ž, Riccardi G, Taskinen MR, Tokgozoglu L, Verschuren WMM, Vlachopoulos C, Wood DA, Zamorano JL, Cooney MT, ESC Scientific Document Group (2016) 2016 ESC/EAS guidelines for the management of dyslipidaemias. Eur Heart $\mathbf{J}$ 37(39):2999-3058. https://doi.org/10.1093/eurheartj/ehw272

2. Aboyans V, Ricco JB, Bartelink MEL, Björck M, Brodmann M, Cohnert T, Collet JP, Czerny M, De Carlo M, Debus S, EspinolaKlein C, Kahan T, Kownator S, Mazzolai L, Naylor AR, Roffi M, Röther J, Sprynger M, Tendera M, Tepe G, Venermo M, Vlachopoulos C, Desormais I, ESC Scientific Document Group (2018) 2017 ESC guidelines on the diagnosis and treatment of peripheral arterial diseases, in collaboration with the European Society for Vascular Surgery (ESVS): document covering atherosclerotic disease of extracranial carotid and vertebral, mesenteric, renal, upper and lower extremity arteries. Eur Heart J 39(9):763-816. https://doi.org/10.1093/eurheartj/ehx095 (Endorsed by: the European Stroke Organization (ESO),The Task Force for the Diagnosis and Treatment of Peripheral Arterial Diseases of the European Society of Cardiology (ESC) and of the European Society for Vascular Surgery (ESVS)
3. Gerhard-Herman MD, Gornik HL, Barrett C, Barshes NR, Corriere MA, Drachman DE, Fleisher LA, Fowkes FG, Hamburg NM, Kinlay S, Lookstein R, Misra S, Mureebe L, Olin JW, Patel RA, Regensteiner JG, Schanzer A, Shishehbor MH, Stewart KJ, Treat-Jacobson D, Walsh ME (2017) 2016 AHA/ACC guideline on the management of patients with lower extremity peripheral artery disease: executive summary: a report of the American College of Cardiology/ American Heart Association task force on clinical practice guidelines. Circulation 135(12):e686-e725. https://doi.org/10.1161/CIR. 0000000000000470

4. Lawall H, Huppert P, Espinola-Klein C, Zemmrich CS, Ruemenapf $G$ (2017) German guideline on the diagnosis and treatment of peripheral artery disease-a comprehensive update 2016. Vasa 46(2):79-86. https://doi.org/10.1024/0301-1526/a000603

5. Norgren L, Hiatt WR, Dormandy JA, Nehler MR, Harris KA, Fowkes FG, Bell K, Caporusso J, Durand-Zaleski I, Komori K, Lammer J, Liapis C, Novo S, Razavi M, Robbs J, Schaper N, Shigematsu H, Sapoval M, White C, White J, Clement D, Creager M, Jaff M, Mohler E 3rd, Rutherford RB, Sheehan P, Sillesen H, Rosenfield K, TASC II Working Group (2007) Inter-society consensus for the management of peripheral rterial disease (TASC II). Eur J Vasc Endovasc Surg 33(Suppl 1):S1-S75

6. Kelly E, Hemphill L (2017) Lipoprotein(a): A lipoprotein whose time has come. Curr Treat Options Cardiovasc Med 19(7):48. https://doi.org/10.1007/s11936-017-0549-z

7. Zeymer U, Parhofer KG, Pittrow D, Binz C, Schwertfeger M, Limbourg T, Röther J (2009) Risk factor profile, management and prognosis of patients with peripheral arterial disease with or without coronary artery disease: results of the prospective German REACH registry cohort. Clin Res Cardiol 98(4):249-256. https://doi.org/10.1007/s00392-009-0754-1

8. Pereg D, Elis A, Neuman Y, Mosseri M, Segev D, Granek-Catarivas M, Lishner M, Hermoni D (2014) Comparison of mortality in patients with coronary or peripheral artery disease following the first vascular intervention. Coron Artery Dis 25(1):79-82. https:// doi.org/10.1097/MCA.0000000000000044

9. Bonaca MP, Nault P, Giugliano RP, Keech AC, Pineda AL, Kanevsky E, Kuder J, Murphy SA, Jukema JW, Lewis BS, Tokgozoglu L, Somaratne R, Sever PS, Pedersen TR, Sabatine MS (2018) Low-density lipoprotein cholesterol lowering with Evolocumab and outcomes in patients with peripheral artery disease: insights from the FOURIER Trial (further cardiovascular outcomes research with PCSK9 inhibition in subjects with elevated risk). Circulation 137(4):338-350. https://doi.org/10.1161/CIRCULATIONAHA. 117.032235

10. Bonaca MP, Bhatt DL, Storey RF, Steg PG, Cohen M, Kuder J, Goodrich E, Nicolau JC, Parkhomenko A, López-Sendón J, Dellborg M, Dalby A, Špinar J, Aylward P, Corbalán R, Abola MTB, Jensen EC, Held P, Braunwald E, Sabatine MS (2016) Ticagrelor for prevention of Ischemic Eventas after myocardial infarction in patients with peripheral artery disease. J Am Coll Cardiol 67(23):2719-2728. https://doi.org/10.1016/j.jacc.2016.03. 524

11. Dieplinger B, Lingenhel A, Baumgartner N, Poelz W, Dieplinger H, Haltmayer M, Kronenberg F, Mueller T (2007) Increased serum lipoprotein(a) concentrations and low molecular weight phenotypes of apolipoprotein(a) are associated with symptomatic peripheral arterial disease. Clin Chem 53(7):1298-1305 (Jul)

12. Laschkolnig A, Kollerits B, Lamina C, Meisinger C, Rantner B, Stadler M, Peters A, Koenig W, Stöckl A, Dähnhardt D, Böger CA, Krämer BK, Fraedrich G, Strauch K, Kronenberg F (2014) Lipoprotein (a) concentrations, apolipoprotein (a) phenotypes, and peripheral arterial disease in three independent cohorts. Cardiovasc Res 103(1):28-36. https://doi.org/10.1093/cvr/cvu107

13. Bérard AM, Bedel A, Le Trequesser R, Freyburger G, Nurden A, Colomer S, Guérin V, Vergnes MC, Becker F, Camelot G, Bres- 
solette L, Lacroix P, Cambou JP, Bura-Rivière A, Emmerich J, Darmon M, Deletraz AM, Mesli S, Colombies B, Vanbrugghe V, Conri C, Constans J (2013) Novel risk factors for premature peripheral arterial occlusive disease in non-diabetic patients: a casecontrol study. PLoS ONE 8(3):e37882. https://doi.org/10.1371/ journal.pone.0037882

14. Forbang NI, Criqui MH, Allison MA, Ix JH, Steffen BT, Cushman M, Tsai MY (2016) Sex and ethnic differences in the associations between lipoprotein(a) and peripheral arterial disease in the Multi-Ethnic Study of Atherosclerosis. J Vasc Surg 63(2):453-458. https://doi.org/10.1016/j.jvs.2015.08.114

15. Gurdasani D, Sjouke B, Tsimikas S, Hovingh GK, Luben RN, Wainwright NW, Pomilla C, Wareham NJ, Khaw KT, Boekholdt SM, Sandhu MS (2012) Lipoprotein(a) and risk of coronary, cerebrovascular, and peripheral artery disease: the EPIC-Norfolk prospective population study. Arterioscler Thromb Vasc Biol 32(12):3058-3065. https://doi.org/10.1161/ATVBAHA.112. 255521

16. Tunstall-Pedoe H, Peters SAE, Woodward M, Struthers AD, Belch JJF (2017) Twenty-year predictors of peripheral arterial disease compared with coronary heart disease in the Scottish Heart Health Extended Cohort (SHHEC). J Am Heart Assoc 6(9):e5967. https:// doi.org/10.1161/JAHA.117.005967

17. Volpato S, Vigna GB, McDermott MM, Cavalieri M, Maraldi C, Lauretani F, Bandinelli S, Zuliani G, Guralnik JM, Fellin R, Ferrucci $L$ (2010) Lipoprotein(a), inflammation, and peripheral arterial disease in a community-based sample of older men and women (the InCHIANTI study). Am J Cardiol 105(12):1825-1830. https://doi. org/10.1016/j.amjcard.2010.01.370

18. Sanchez Muñoz-Torrero JF, Rico-Martín S, Álvarez LR, Aguilar E, Alcalá JN, Monreal M, FRENA Investigators (2018) Lipoprotein (a) levels and outcomes in stable outpatients with symptomatic artery disease. Atherosclerosis 276:10-14. https://doi.org/10.1016/ j.atherosclerosis.2018.07.001

19. Tsimikas S (2018) RNA-targeted therapeutics for lipid disorders. Curr Opin Lipidol 29(6):459-466. https://doi.org/10.1097/MOL. 0000000000000549

20. Jaeger BR, Richter Y, Nagel D, Heigl F, Vogt A, Roeseler E, Parhofer K, Ramlow W, Koch M, Utermann G, Labarrere CA, Seidel D, Group of Clinical Investigators (2009) Longitudinal cohort study on the effectiveness of lipid apheresis treatment to reduce high lipoprotein(a) levels and prevent major adverse coronary events. Nat Clin Pract Cardiovasc Med 6(3):229-239. https://doi. org/10.1038/ncpcardio1456

21. Leebmann J, Roeseler E, Julius U, Heigl F, Spitthoever R, Heutling D, Breitenberger P, Maerz W, Lehmacher W, Heibges A, Klingel R, Pro(a)LiFe Study Group (2013) Lipoprotein apheresis in patients with maximally tolerated lipid-lowering therapy, lipoprotein(a)-hyperlipoproteinemia, and progressive cardiovascular disease: prospective observational multicenter study. Circulation 128(24):2567-2576. https://doi.org/10.1161/CIRCULATIONAHA. 113.002432

22. Roeseler E, Julius U, Heigl F, Spitthoever R, Heutling D, Breitenberger P, Leebmann J, Lehmacher W, Kamstrup PR, Nordestgaard BG, Maerz W, Noureen A, Schmidt K, Kronenberg F, Heibges A, Klingel R, Pro(a)LiFe-Study Group (2016) Lipoprotein apheresis for lipoprotein(a)-associated cardiovascular disease: prospective 5 years of follow-up and apolipoprotein(a) characterization. Arterioscler Thromb Vasc Biol 36(9):2019-2027. https://doi.org/10. 1161/ATVBAHA.116.307983

23. Schatz U, Tselmin S, Müller G, Julius U, Hohenstein B, Fischer S, Bornstein SR (2017) Most significant reduction of cardiovascular events in patients undergoing lipoproteinapheresis due to raised Lp(a) levels-a multicenter observational study. Atheroscler Suppl 30:246-252. https://doi.org/10.1016/j.atherosclerosissup.2017.05. 047

24. Schampera S, Fischer S, Weiss N, Julius U (2015) Detailed description of the cardiovascular situation in patients who have started lipoprotein apheresis treatment. Atheroscler Suppl 18:209-214. https://doi.org/10.1016/j.atherosclerosissup.2015.02.031

25. Heigl F, Hettich R, Lotz N, Reeg H, Pflederer T, Osterkorn D, Osterkorn K, Klingel R (2015) Efficacy, safety, and tolerability of long-term lipoprotein apheresis in patients with LDL- or Lp(a) hyperlipoproteinemia: Findings gathered from more than 36,000 treatments at one center in Germany. Atheroscler Suppl 18:154-162. https://doi.org/10.1016/j.atherosclerosissup.2015.02.013

26. Poller WC, Dreger H, Morgera S, Berger A, Flessenkämper I, Enke-Melzer K (2015) Lipoprotein apheresis in patients with peripheral artery disease and hyperlipoproteinemia(a). Atheroscler Suppl 18:187-193. https://doi.org/10.1016/j.atherosclerosissup. 2015.02.032

27. Poller WC, Berger A, Dreger H, Morgera S, Enke-Melzer K (2017) Lipoprotein apheresis in patients with peripheral artery disease and lipoprotein(a)-hyperlipoproteinemia: 2-year follow-up of a prospective single center study. Atheroscler Suppl 30:174-179. https://doi.org/10.1016/j.atherosclerosissup.2017.05.007

28. Julius U (2017) Current role of lipoprotein apheresis in the treatment of high-risk patients. J Cardiovasc Dev Dis 5(2):E27. https:// doi.org/10.3390/jcdd5020027

29. Hohenstein B, Julius U, Lansberg P, Jaeger B, Mellwig KP, Weiss N, Graehlert X, Roeder I, Ramlow W (2017) Rationale and design of MultiSELECt: A European Multicenter Study on the Effect of Lipoprotein(a) Elimination by lipoprotein apheresis on Cardiovascular outcomes. Atheroscler Suppl30:180-186. https://doi.org/10. 1016/j.atherosclerosissup.2017.05.009 